\title{
Docetaxel-Induced Peripheral Neuropathy in Breast Cancer Patients Treated with Adjuvant or Neo-Adjuvant Chemotherapy
}

\author{
Hui Lin Cheng ${ }^{a} \quad$ Alex Molassiotis $^{a} \quad$ Anthony Kwun To Leung ${ }^{b}$ Kam Hung Wong ${ }^{b}$ \\ ${ }^{a}$ School of Nursing, The Hong Kong Polytechnic University, Hong Kong, Hong Kong SAR; ${ }^{b}$ Department of Clinical \\ Oncology, Queen Elizabeth Hospital, Hong Kong, Hong Kong SAR
}

\section{Keywords}

Breast cancer · Chemotherapy · Docetaxel · Peripheral neuropathy $\cdot$ Health-related quality of life

\begin{abstract}
Background: Docetaxel-induced peripheral neuropathy (PN) is typically manifested as sensory and motor neuropathy. This study aimed to investigate the incidence, duration, and risk factors of sensory and motor $\mathrm{PN}$ and their impact on health-related quality of life (HRQOL) among breast cancer (BC) patients during the first year since starting docetaxelbased chemotherapy. Methods: We reported a secondary analysis of longitudinal data on docetaxel-induced PN and HRQOL among 127 BC patients. Results: Cumulative incidence rates of motor and sensory PN were 31.5 and $21.3 \%$, while the median durations of motor and sensory PN were 6 and 13 weeks. A consistently significant risk factor for both PNs was a cumulative docetaxel dose of $>300 \mathrm{mg} / \mathrm{m}^{2}$. A significant interaction between sensory PN and time was found for physical and social functioning, while a significant motor $\mathrm{PN}$ and time interaction effect was identified for physical functioning only. Conclusions: Motor PN was more common than sensory PN in BC patients treated with docetaxel. Both types of PN had a significant impact on physical functioning.
\end{abstract}

(c) 2020 S. Karger AG, Basel

\section{Introduction}

Breast cancer (BC) remains the most frequently diagnosed cancer among women worldwide. Women with invasive $\mathrm{BC}$ are usually treated with taxanes, primarily paclitaxel and docetaxel. These chemotherapeutic agents can cause peripheral neuropathy $(\mathrm{PN})$ that is typically manifested as sensory (e.g., numbness in the fingers/ toes) and motor (e.g., muscle weakness) neuropathy that may be accompanied with autonomic symptoms [1]. The occurrence of PN has been found to impact the patients' health-related quality of life (HRQOL) and in some patients, resulting in chemotherapy dose delay or reduction $[2,3]$. Although extensive evidence has revealed the distinct clinical patterns of motor and sensory PN caused by paclitaxel, limited research is available on docetaxel-induced PN. Docetaxel-associated PN can occur during cycles of chemotherapy but may persist up to 13 years following treatment completion [4]. Its incidence can vary from 11 to $64 \%$, depending on the assessment point during or after the treatment [5]. Risk factors for docetaxel-induced PN have been inconsistently identified, including older age ( $\geq 55$ years), diabetes, a cumulative docetaxel dose of $>300 \mathrm{mg} / \mathrm{m}^{2}$, docetaxel protocol and dietary factors [3, 6-7]. This paper aimed to investigate the incidence, duration, and risk factors of sensory and motor PN and their impact on HRQOL among BC patients during the first year since starting docetaxel-based chemotherapy.

\section{Methods}

Design and Setting

The parent study was a large multinational, prospective longitudinal study examining the progression of chemotherapy-induced PN, while the key findings on prevalence, risk factors and measurement of PN have been reported elsewhere [8-10]. We reported a secondary analysis of longitudinal data from the parent 
study on PN and HRQOL among 127 BC patients who received at least one cycle of docetaxel-based chemotherapy at Queen Elizabeth Hospital in Hong Kong. The inclusion criteria were: (a) diagnosis of BC; (b) female adult (>18 years old); (c) chemotherapy naïve; (d) about to receive docetaxel-based chemotherapy; and (e) expected survival of at least 12 months as determined by oncologists. Patients were excluded from this study if they (a) presented with preexisting PN due to any cause (researcher-rated NCI-CT$\mathrm{CAE} \geq 2$ ) before starting chemotherapy or (b) discontinued chemotherapy after the first cycle.

\section{Data Collection}

PN and HRQOL were assessed from baseline (pre-chemotherapy), after each chemotherapy cycle (maximum 6 cycles, 3 weeks per cycle), and at 6, 9, and 12 months after starting chemotherapy. $\mathrm{PN}$ was assessed using the NCI-CTCAE and the traditional Chinese version of the European Organization for Research and Treatment of Cancer Quality of Life-Chemotherapy-Induced Peripheral Neuropathy twenty-item Questionnaire (EORTC QLQCIPN20) $[10,11]$. The NCI-CTCAE is a clinician-based grading scale for PN and consists of two items: sensory and motor PN. Each item is graded from 1 (asymptomatic) to 5 (death), with a higher grade representing greater $\mathrm{PN}$ [11]. Evidence suggests that the inter-rater reliability of the NCI-CTCAE after training is moderate (weighted Cohen's $\kappa$ coefficient $>0.7$ ) [12]. For this study, we used $10 \mathrm{~g}$ monofilament assessment and physical examination (e.g., tendon reflexes) before a score on the NCI-CTCAE was assigned. The inter-rater reliability of the NCI-CTCAE after training was 0.8 . The EORTC QLQ-CIPN20 questionnaire has 20 items and in addition to sensory and motor subscales, it also assesses autonomic symptoms [13]. It is rated on a 4-point Likert scale from 1 to 4 , and a score of $\geq 2$ indicates the presence of a symptom. Only the five functioning scales (physical-, role-, emotional-, cognitive-, and social functioning) and the global QOL scale were used for this study [14]. Demographic and clinical data were extracted from medical records. The following information was collected: age, cancer stage, diabetes, and chemotherapy characteristics such as treatment intention and regimen, number of cycles of chemotherapy received, cumulative dosage, and presence of chemotherapy reduction or delays due to PN. Habitual data were gathered from patients, which included smoking history, alcohol intake, and vegetable intake.

\section{Statistical Analysis}

Data were analyzed using SPSS 22.0. Descriptive statistics were used to describe the sample. Kaplan-Meier methods were employed to estimate the cumulative incidence and duration of motor and sensory PN. Cox proportional hazards regression models were used to identify risk factors that were related with motor and sensory PN. Generalized estimation equations (GEEs) were used to analyze changes in the severity of individual sensory, motor, and autonomic PN symptoms and HRQOL over time. To investigate the impact of PN on HRQOL, patients were stratified by duration of PN: (a) never experienced PN, (b) sporadic PN, and (c) persistent PN. GEE models were used to investigate PN-time interaction effects. The level of significance was set at 0.05 (2-sided).

\section{Results}

\section{Patient Characteristics}

The 127 patients analyzed (Table 1 ) were 51.9 years old on average, and $35.4 \%$ were aged less than 55 years. The
Table 1. Participant characteristics $(n=127)$

\begin{tabular}{|c|c|}
\hline Age, years & $51.9 \pm 8.0$ \\
\hline$\leq 55$ years & $82(64.6)$ \\
\hline$>55$ years & $45(35.4)$ \\
\hline \multicolumn{2}{|l|}{ Cancer stage } \\
\hline I & $34(26.8)$ \\
\hline II & $60(47.2)$ \\
\hline III $^{\mathrm{a}}$ & $33(26.0)$ \\
\hline \multicolumn{2}{|l|}{ Treatment intent } \\
\hline Adjuvant & $103(81.1)$ \\
\hline Neoadjuvant & $24(18.9)$ \\
\hline \multicolumn{2}{|l|}{ Chemotherapy protocol } \\
\hline Adriamycin + cyclophosphamide to docetaxel & $15(11.8)$ \\
\hline Docetaxel + cyclophosphamide & $65(51.2)$ \\
\hline Docetaxel + adriamycin + cyclophosphamide & $19(15.0)$ \\
\hline Docetaxel +carboplatin + herceptin & $28(22.0)$ \\
\hline Cycles of docetaxel treatment ( 3 weeks/cycle), $n$ & $5.1 \pm 1.5$ \\
\hline Cumulative dosage, $\mathrm{mg} / \mathrm{m}^{2}$ & $357.9 \pm 82.2$ \\
\hline$\leq 300 \mathrm{mg} / \mathrm{m}^{2}$ & $64(49.6)$ \\
\hline$>300 \mathrm{mg} / \mathrm{m}^{2}$ & $65(50.4)$ \\
\hline \multicolumn{2}{|l|}{ Diabetes } \\
\hline No & $112(88.2)$ \\
\hline Yes & $15(11.8)$ \\
\hline \multicolumn{2}{|l|}{ Smoking history } \\
\hline Ex-smoker & $10(7.9)$ \\
\hline Never smoker & $117(92.1)$ \\
\hline \multicolumn{2}{|l|}{ Alcohol intake per day ${ }^{b}$} \\
\hline$<1$ & $122(96.1)$ \\
\hline$>1$ & $5(3.9)$ \\
\hline \multicolumn{2}{|l|}{ Vegetable intake per day } \\
\hline 1 serving or none & $62(48.8)$ \\
\hline$\geq 2$ servings & $65(51.2)$ \\
\hline
\end{tabular}

Data are presented as mean $\pm \mathrm{SD}$ or $n(\%)$. ${ }^{\text {a }}$ Contains 2 cases with stage IV. ${ }^{\mathrm{b}}$ One standard drink containing alcohol.

mean cumulative docetaxel dosage was $357.9 \mathrm{mg} / \mathrm{m}^{2}$. No chemotherapy dose reduction and delay due to PN occurred in this sample.

\section{Cumulative Incidence and Duration of Motor and Sensory PN (NCI-CTCAE Scale)}

Cumulative incidence rates of motor and sensory PN were 31.5 and $21.3 \%$, while the median durations of motor and sensory PN were 6 weeks (range $=0-43$ ) and 13 weeks (range $=0-40$ ), respectively. (Fig. 1).

\section{Prevalence and Severity of Motor, Sensory, and Autonomic PN Symptoms (EORTC QOL-CIPN20 Scale)}

For motor symptoms, the peak prevalence rates were between $7.2 \%$ ("problem in holding a pen") and 26.9\% ("difficulty in climbing stairs or getting up out of a chair because of weakness in legs"). For sensory symptoms, the peak prevalence rates varied from $5.4 \%$ ("difficulty distinguishing between hot and cold water") to $43.5 \%$ ("numbness in fingers or hands"). Regarding autonomic symp- 
Table 2. Risk factors for motor and sensory peripheral neuropathy $(n=127)$

\begin{tabular}{|c|c|c|c|c|c|c|c|c|c|c|}
\hline \multirow[t]{2}{*}{ Risk factors } & \multicolumn{5}{|c|}{ Motor PN } & \multicolumn{5}{|c|}{ Sensory PN } \\
\hline & $\begin{array}{l}\text { present, } \\
n(\%)\end{array}$ & $\begin{array}{l}\text { absent, } \\
n(\%)\end{array}$ & HR & $95 \% \mathrm{CI}$ & $p$ value & $\begin{array}{l}\text { present, } \\
n(\%)\end{array}$ & $\begin{array}{l}\text { absent, } \\
n(\%)\end{array}$ & HR & $95 \% \mathrm{CI}$ & $p$ value \\
\hline \multicolumn{11}{|c|}{ Type of docetaxel-based chemotherapy } \\
\hline $\mathrm{AC} \rightarrow \mathrm{T}$ & $6(15.0)$ & $9(10.3)$ & 1.00 & & & $5(18.5)$ & $10(10.0)$ & 1.00 & & \\
\hline TAC & $5(12.5)$ & $14(16.1)$ & 0.70 & $0.21-2.30$ & 0.14 & $3(11.1)$ & $16(16.0)$ & 0.50 & $0.12-2.11$ & 0.34 \\
\hline $\mathrm{TCH}$ & $7(17.5)$ & $21(24.1)$ & 0.65 & $0.22-2.00$ & 0.55 & $3(11.1)$ & $25(25.0)$ & 0.32 & $0.08-1.40$ & 0.13 \\
\hline $\mathrm{TC}$ & $22(55.0)$ & $43(49.4)$ & 2.74 & $0.70-10.76$ & 0.46 & $16(59.3)$ & $49(49.0)$ & 4.27 & $0.81-22.60$ & 0.09 \\
\hline \multicolumn{11}{|c|}{ Cumulative dose of docetaxel } \\
\hline$\leq 300 \mathrm{mg} / \mathrm{m}^{2}$ & $19(47.5)$ & $45(51.7)$ & 1.00 & & & $13(48.1)$ & $51(51.0)$ & 1.00 & & \\
\hline$>300 \mathrm{mg} / \mathrm{m}^{2}$ & $21(52.5)$ & $42(48.3)$ & 3.58 & $1.10-11.64$ & $<0.05$ & $14(51.9)$ & $49(49.0)$ & 7.20 & $1.70-30.53$ & $<0.01$ \\
\hline \multicolumn{11}{|l|}{ Diabetes } \\
\hline No & $34(85.0)$ & $78(89.7)$ & 1.00 & & & $23(85.2)$ & $89(89.0)$ & 1.00 & & \\
\hline Yes & $6(15.0)$ & $9(10.3)$ & 1.18 & $0.47-3.00$ & 0.72 & $4(14.8)$ & $11(11.0)$ & 1.06 & $0.32-3.50$ & 0.93 \\
\hline \multicolumn{11}{|l|}{ Age } \\
\hline$\leq 55$ years & $19(47.5)$ & $63(72.4)$ & 1.00 & & & $14(51.9)$ & $68(68.0)$ & 1.00 & & \\
\hline$>55$ years & $21(52.5)$ & $24(27.6)$ & 2.02 & $1.03-3.97$ & $<0.05$ & $13(48.1)$ & $32(32.0)$ & 1.42 & $0.61-3.29$ & 0.41 \\
\hline \multicolumn{11}{|c|}{ Vegetable intake per day } \\
\hline$\leq 1$ serving & $21(52.5)$ & $41(47.1)$ & 1.00 & & & $15(55.6)$ & $47(47.0)$ & 1.00 & & \\
\hline$\geq 2$ servings & $19(47.5)$ & $46(52.9)$ & 0.85 & $0.44-1.64$ & 0.66 & $12(44.4)$ & $53(53.0)$ & 0.72 & $0.31-1.67$ & 0.44 \\
\hline
\end{tabular}

$\mathrm{HR}$, hazard ratio; $\mathrm{AC} \rightarrow \mathrm{T}$, adriamycin + cyclophosphamide to docetaxel; $\mathrm{TAC}$, docetaxel + adriamycin + cyclophosphamide; TCH, docetaxel + carboplatin + herceptin; TC, docetaxel + cyclophosphamide; PN, peripheral neuropathy.

toms, the peak prevalence rate was $14.8 \%$ ("dizzy when standing up from a sitting or lying position") and 31.1\% ("blurred vision"), respectively.

GEE analyses demonstrated significant changes in the severity over time for five out of the seven motor symptoms $(p<0.05-0.001)$ : including "problems in holding a pen, which made writing difficult," "difficulty manipulating small objects with fingers," "difficulty opening a jar or bottle because of weakness in hands," "difficulty in walking because feet dropped downward," and "difficulty in climbing stairs or getting up out of a chair because of weakness in legs." Significant longitudinal changes in the severity were observed for six out of the nine motor symptoms $(p<0.05-0.001)$ : including "tingling in fingers/hands," "tingling in toes/feet," "numbness in fingers or hands" $(p<0.001)$, "numbness in toes or feet," "problems in standing or walking because of difficulty feeling the ground under feet," and "difficulty in distinguishing between hot and cold water." For autonomic symptoms, only the symptom of "dizzy when standing up from a sitting or lying position" $(p<0.05)$ showed a significant change in the severity over time. The prevalence and severity of motor, sensory, and autonomic PN symptoms over time are shown in supplementary file 1 (see www. karger.com/doi/10.1159/000507843 for all suppl. material).

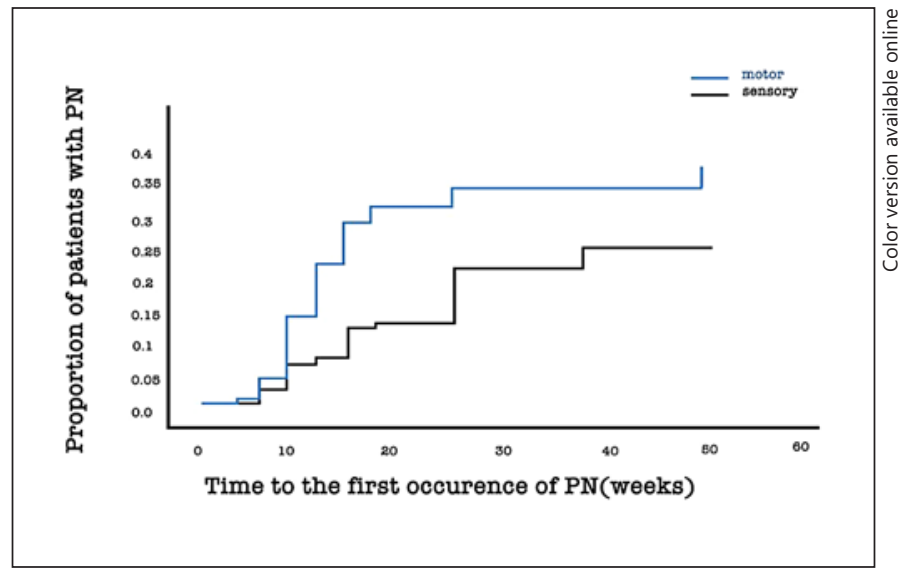

Fig. 1. Cumulative incidence of motor and sensory peripheral neuropathy $(\mathrm{PN})$ in patients treated with docetaxel.

\section{Risk Factors of Motor and Sensory PN}

In Table 2, two significant risk factors were identified for motor PN, including a cumulative dose of docetaxel $>$ $300 \mathrm{mg} / \mathrm{m}^{2}(\mathrm{HR}=3.58,95 \%$ CI $1.10-11.64)$ and age $>55$ $(\mathrm{HR}=2.02,95 \% \mathrm{CI} 1.03-3.97)$. The only significant risk factor for sensory PN was a cumulative dose of docetaxel $>300 \mathrm{mg} / \mathrm{m}^{2}$ only $(\mathrm{HR}=7.20,95 \%$ CI $1.70-30)$. 


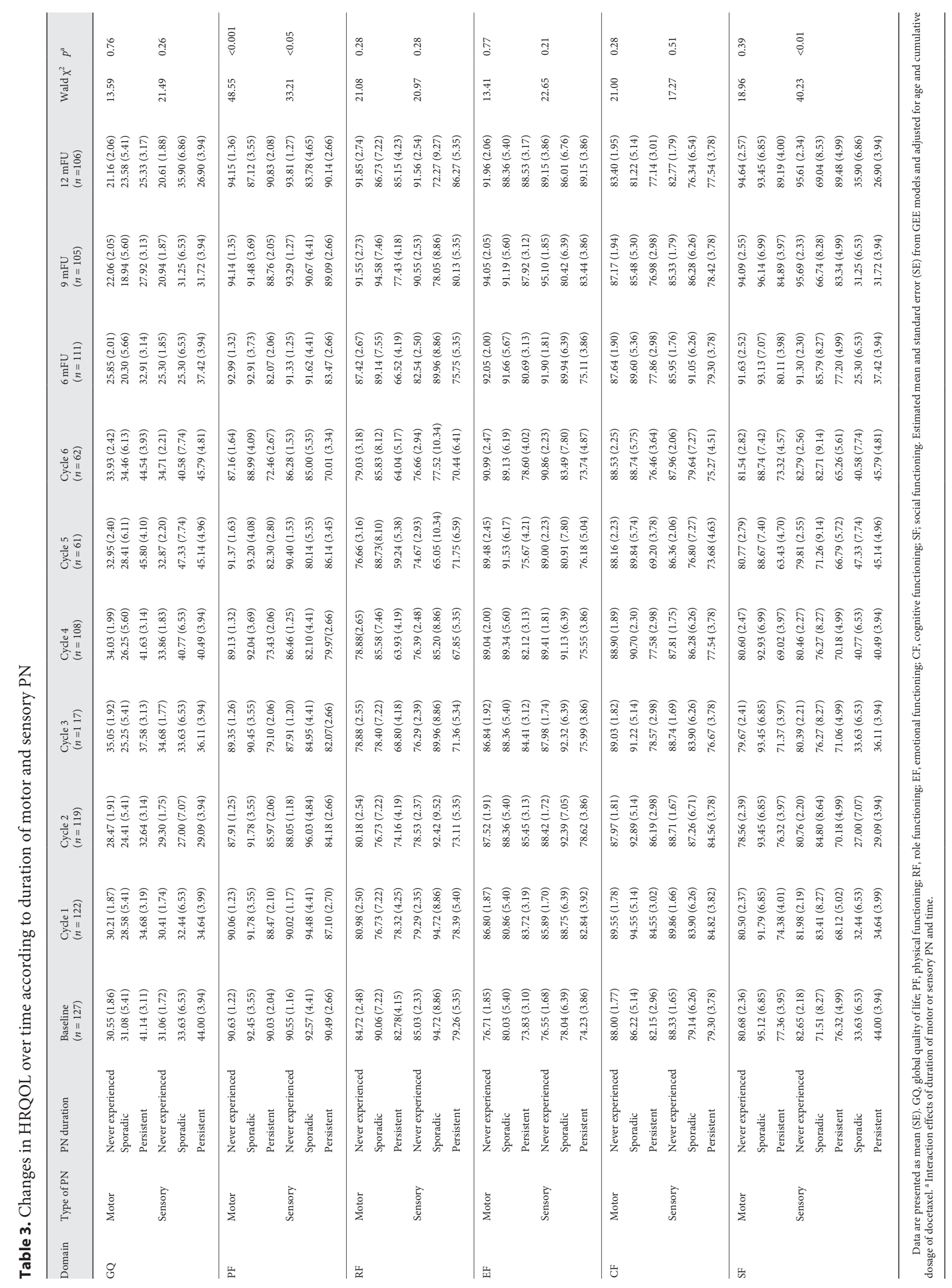



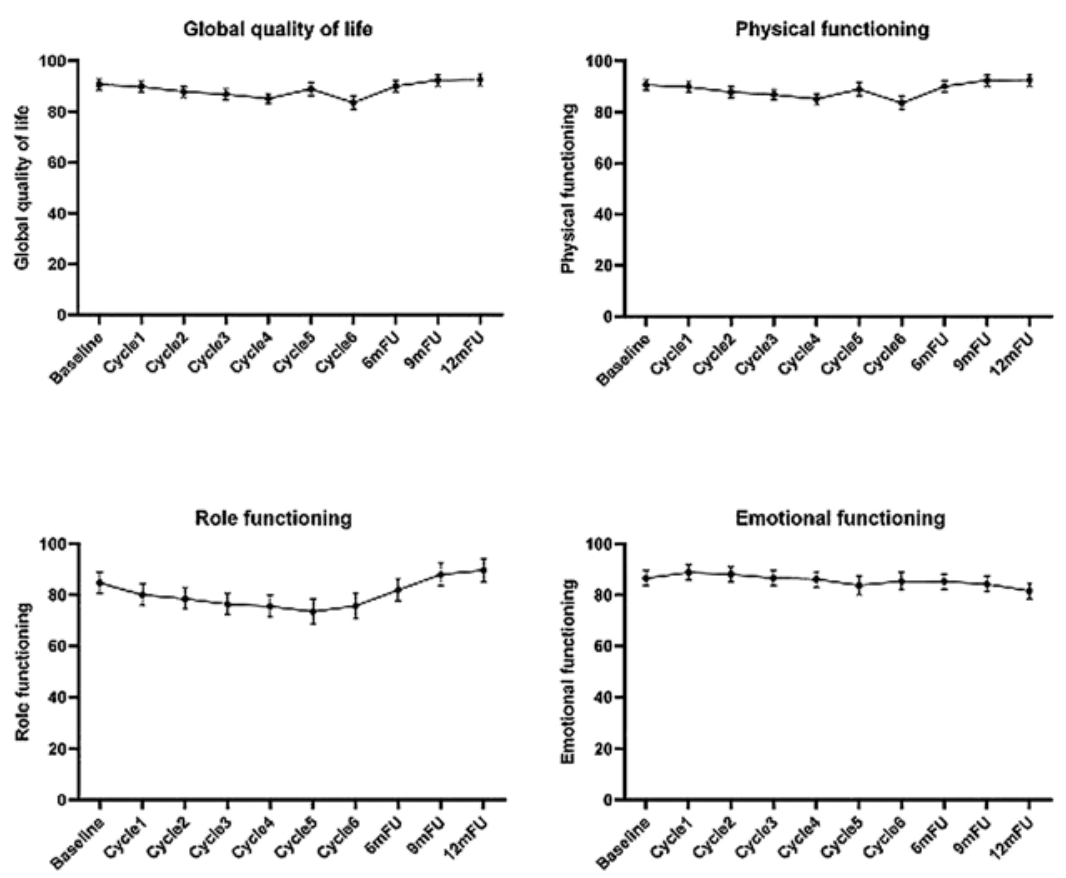

Fig. 2. Course of health-related quality of life (estimated mean and 95\% CI) in breast cancer patients treated with docetaxel over time.
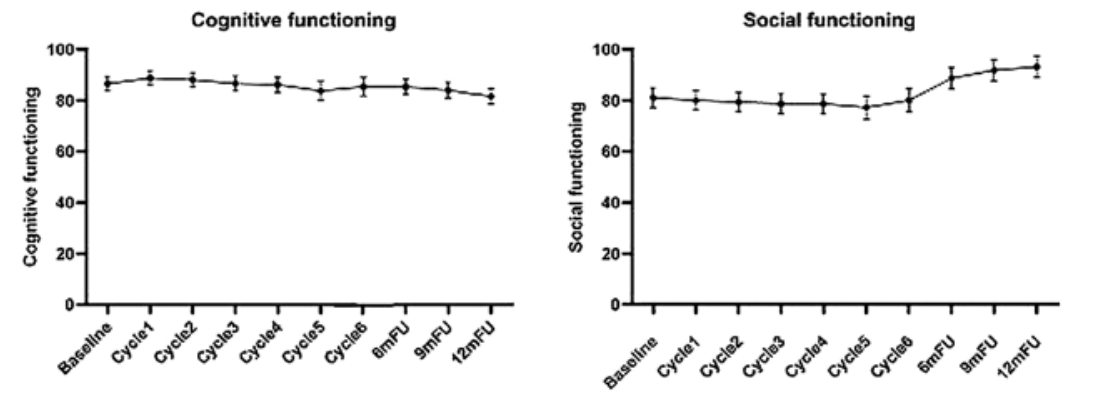

Motor/Sensory PN and HRQOL

According to GEE analyses, HRQOL in all domains significantly changed over time (all $p<0.001$ ) except for cognitive functioning (Fig. 2). However, when considering the impact of motor PN on HRQOL, a statistically significant interaction effect (Wald $\chi^{2}=48.55, p<0.001$ ) between motor PN and time was found for physical functioning only. Regarding sensory PN, there were statistically significant interactions with time for physical functioning (Wald $\chi^{2}=33.21, p<0.05$ ) and social functioning (Wald $\chi^{2}=40.23, p<0.01$ ) (Table 3).

\section{Discussion}

This study is one of the few studies investigating motor and sensory PN induced by docetaxel. Overall, the cumulative incidences of motor and sensory $\mathrm{PN}$ observed in this study have been found to fall within the range of data from the National Comprehensive Cancer Network Task Force Report (11-64\%), although the report has not specified the type of PN [5]. Recent data have also showed that the incidence of docetaxel-induced PN can vary from 15 to $41.9 \%$ in $\mathrm{BC}$ patients depending on different measures of PN $[6,7]$.

Yet, it is interesting to observe that the cumulative incidence of motor PN (31.5\%) was higher than that of sensory PN $(21.3 \%)$. This finding is inconsistent with the seemingly well-established knowledge on nature of PN caused by taxane, with $\mathrm{PN}$ being characterized by sensory neuropathy and less so with motor neuropathy [2]. Nevertheless, a small-scale study of $\mathrm{BC}$ patients receiving docetaxel reported that motor symptoms including muscle weakness and trouble with balance were more frequently reported than sensory symptoms such as numbness, tingling, and cold sensitivity [15]. While no subse- 
quent study has been conducted to explain docetaxel-associated motor symptoms, the reason underlying this unique finding is still unknown and future investigation is warranted.

Regarding motor symptoms, our study found that cramps in the legs/hands did not demonstrate a significant change in the severity over time. In a large study of 1,031 early BC patients who received at least one cycle of docetaxel, it was found that cramps in the legs was the most frequently reported symptom of PN [6]. As this study did not collect the baseline data, changes in this symptom over time could not be observed. The present study finding can be explained by the fact that cramps in the legs/hands may not represent a symptom of motor PN due to docetaxel itself but are related to patients' age or preexisting comorbid conditions.

Risk factors for docetaxel-induced PN remain unclear as studies in the literature have yielded conflicting results. We found that both PNs were significantly predicted by a cumulative dose of $>300 \mathrm{mg} / \mathrm{m}^{2}$, and this is consistent with a study [15]. However, the present study did not find any relationship between a particular docetaxel regimen and PN possibly due to acknowledging the small sample size of these chemotherapy subgroups for a more concrete analysis [7].

Although old age is found as a known risk factor for PN caused by taxane or docetaxel $[6,7]$, notably in this sample, older patients reported a higher risk of motor PN. Accumulating evidence indicates that $\mathrm{PN}$ is associated with a risk of fall in BC patients [7]. This is likely explained by muscle weakness and loss of balance affecting the lower extremities, which are major clinical features of motor PN. Our finding suggests the importance of observing the occurrence and progression of motor $\mathrm{PN}$ in older BC patients treated with docetaxel.

We also found that vegetable intake was not a significant risk factor of $\mathrm{PN}$, and this finding is consistent with a recent finding from a study of 1,237 BC patients in United States reporting no association between vegetable intake and PN [16]. Furthermore, studies on whether diabetic patients are more predisposed to the development of PN after chemotherapy are also inconclusive $[17,18]$. Indeed, in this present study, no significant relationship between diabetes and $\mathrm{PN}$ was found.

This study also showed statistically significant interaction effects of motor/sensory PN-time on physical and social functioning at specific time points. These results appear to be consistent with a systematic review and recent studies on the relationship of PN and HRQOL among cancer patients receiving chemotherapy in general or BC patients receiving docetaxel $[2,6]$. However, it is important to note that motor/sensory PN consistently and greatly impacted physical functioning, which is a key domain of HRQOL.
The strength of this study lies in combining the NCICTACE and EORTC CIPN-20 for offering a broad view of motor and sensory PN symptoms due to docetaxel. The NCI-CTCAE has been criticized for underestimating the incidence and severity of PN due to its suboptimal interrater reliability in identifying and quantifying PN [19]. To overcome such limitation, only one researcher after training was invited to assess $\mathrm{PN}$ with the assistance of objective tests (e.g., $10 \mathrm{~g}$ monofilament) [20]. As there is no "gold standard" for measuring PN, selection of an acceptable measure with both subjective and objective aspects of PN can be considered to identify PN in future clinical research. Also, as this study focused on the first year from chemotherapy, cumulative incidence and risk factors of both motor and sensory $\mathrm{PN}$ and their impact on HRQOL in BC survivors beyond the first year after treatment initiation is unknown; thus, a follow-up study of PN for a longer duration is suggested.

\section{Conclusion}

This study showed that motor PN was more common than sensory PN, and a cumulative docetaxel dose $>300$ $\mathrm{mg} / \mathrm{m}^{2}$ was a significant risk factor for both PNs in BC patients. Furthermore, the study also found a significant impact of PN on physical functioning. Health professionals should establish awareness of motor $\mathrm{PN}$ as a frequent side effect of docetaxel and inform patients on how to better prepare for PN before starting chemotherapy. Future research should extend to investigate the motor $\mathrm{PN}$ and its associated physical dysfunction in BC patients.

\section{Acknowledgment}

The authors would like to thank all participants for their contribution to the study.

\section{Statement of Ethics}

The study was approved by the Hong Kong Polytechnic University and the Hong Kong Kowloon Central/Kowloon East Research Ethics Committee. Written informed consent was collected from each patient.

\section{Disclosure Statement}

The authors have declared no conflicts of interest. 


\section{Funding Sources}

This study was funded by Departmental General Research Funding of School of Nursing in the Hong Kong Polytechnic University.

\section{Author Contributions}

Conception and design: A.M., H.L.C. Participant recruitment: H.L.C, A.K.T.L., K.H.W. Participant follow-up and assessment: H.L.C. Data analysis: A.M., H.L.C. Contributing to writing the paper including discussion: all. All authors read and approved the final manuscript.

\section{References}

1 De Iuliis F, Taglieri L, Salerno G, Lanza R, Scarpa S. Taxane induced neuropathy in patients affected by breast cancer: literature review. Crit Rev Oncol Hematol. 2015 Oct; 96(1):34-45.

2 Mols F, Beijers T, Vreugdenhil G, van de PollFranse L. Chemotherapy-induced peripheral neuropathy and its association with quality of life: a systematic review. Support Care Cancer. 2014 Aug;22(8):2261-9.

3 Speck RM, Sammel MD, Farrar JT, Hennessy S, Mao JJ, Stineman MG, et al. Impact of chemotherapy-induced peripheral neuropathy on treatment delivery in nonmetastatic breast cancer. J Oncol Pract. 2013 Sep;9(5):e234-40.

4 Osmani K, Vignes S, Aissi M, Wade F, Milani P, Lévy BI, et al. Taxane-induced peripheral neuropathy has good long-term prognosis: a 1- to 13-year evaluation. J Neurol. 2012 Sep; 259(9):1936-43.

5 Stubblefield MD, Burstein HJ, Burton AW, Custodio CM, Deng GE, Ho M, et al. NCCN task force report: management of neuropathy in cancer. J Natl Compr Canc Netw. 2009 Sep; 7 Suppl 5:S1-26.

6 Eckhoff L, Knoop A, Jensen MB, Ewertz M. Persistence of docetaxel-induced neuropathy and impact on quality of life among breast cancer survivors. Eur J Cancer. 2015 Feb; 51(3):292-300.

7 Bandos H, Melnikow J, Rivera DR, Swain SM, Sturtz K, Fehrenbacher L, et al. Long-term peripheral neuropathy in breast cancer patients treated with adjuvant chemotherapy: NRG Oncology/NSABP B-30. J Natl Cancer Inst 2018;110(2):djx162.

8 Molassiotis A, Cheng HL, Lopez V, Au JS, Chan A, Bandla A, et al. Are we mis-estimating chemotherapy-induced peripheral neuropathy? Analysis of assessment methodologies from a prospective, multinational, longitudinal cohort study of patients receiving neurotoxic chemotherapy. BMC Cancer. 2019 Feb;19(1):132.

9 Molassiotis A, Cheng HL, Leung KT, Li YC, Wong $\mathrm{KH}, \mathrm{Au}$ JS, et al. Risk factors for chemotherapy-induced peripheral neuropathy in patients receiving taxane- and platinumbased chemotherapy. Brain Behav. 2019 Jun; 9(6):e01312.

10 Cheng HL, Molassiotis A. Longitudinal validation and comparison of the Chinese version of the European Organization for Research and Treatment of Cancer Quality of Life-Chemotherapy-Induced Peripheral Neuropathy Questionnaire (EORTC QLQ-CIPN20) and the Functional Assessment of Cancer-Gynecologic Oncology Group-Neurotoxicity subscale (FACT/GOG-Ntx). Asia Pac J Clin Oncol. 2019 Feb;15(1):56-62.

11 US Department of Health and Human Services. National Cancer Institute. Common Terminology Criteria for Adverse Events (CTCAE) Version 4.03. 2010 [accessed 2019 June 19]. Available from: https://evs.nci.nih. gov/ftp1/CTCAE/About.html

12 Cavaletti G, Cornblath DR, Merkies IS, Postma TJ, Rossi E, Frigeni B, et al.; CI-PeriNomS Group. The chemotherapy-induced peripheral neuropathy outcome measures standardization study: from consensus to the first validity and reliability findings. Ann Oncol. 2013 Feb;24(2):454-62.

13 Postma TJ, Aaronson NK, Heimans JJ, Muller MJ, Hildebrand JG, Delattre JY, et al. The development of an EORTC quality of life questionnaire to assess chemotherapy-induced peripheral neuropathy: the QLQ-CIPN20. Eur J Cancer 2005;41(8):1135-1139.14.

14 Aaronson NK, Ahmedzai S, Bergman B, Bullinger M, Cull A, Duez NJ, et al. The European
Organization for Research and Treatment of Cancer QLQ-C30: a quality-of-life instrument for use in international clinical trials in oncology. J Natl Cancer Inst. 1993 Mar;85(5): 365-76.

15 Tofthagen C, McAllister RD, Visovsky C. Peripheral neuropathy caused by Paclitaxel and docetaxel: an evaluation and comparison of symptoms. J Adv Pract Oncol 2013;4(4):204215.16.

16 Pereira S, Fontes F, Sonin T, Dias T, Fragoso M, Castro-Lopes JM, et al. Chemotherapyinduced peripheral neuropathy after neoadjuvant or adjuvant treatment of breast cancer: a prospective cohort study. Support Care Cancer. 2016 Apr;24(4):1571-81.

17 Simon NB, Danso MA, Alberico TA, Basch E, Bennett AV. The prevalence and pattern of chemotherapy-induced peripheral neuropathy among women with breast cancer receiving care in a large community oncology practice. Qual Life Res. 2017 Oct;26(10):2763-72.

18 Kus T, Aktas G, Kalender ME, Sevinc A, Kul $\mathrm{S}$, Suner A, et al. Taxane-induced peripheral sensorial neuropathy in cancer patients is associated with duration of diabetes mellitus: a single-center retrospective study. Support Care Cancer. 2016 Mar;24(3):1175-9.

19 Frigeni B, Piatti M, Lanzani F, Alberti P, Villa $\mathrm{P}$, Zanna C, et al. Chemotherapy-induced peripheral neurotoxicity can be misdiagnosed by the National Cancer Institute Common Toxicity scale. J Peripher Nerv Syst. 2011 Sep; 16(3):228-36

20 Bottomley A, Jones D, Claassens L. Patientreported outcomes: assessment and current perspectives of the guidelines of the Food and Drug Administration and the reflection paper of the European Medicines Agency. Eur J Cancer. 2009 Feb;45(3):347-53. 